\title{
Access-related infections in two haemodialysis units: results of a nine-year intervention and surveillance program
}

\author{
Ittamar Gork', Ilana Gross², Matan J. Cohen², Carmela Schwartz², Allon E. Moses², Michal Dranitzki Elhalel ${ }^{1}$ and
} Shmuel Benenson ${ }^{2^{*}}$ (D)

\begin{abstract}
Background: Access-related infections are a major cause of morbidity and mortality in haemodialysis patients. Our goal was to decrease the rate of these infections by implementing an intervention and surveillance program.

Methods: This intervention took place in two haemodialysis units (Units A and B) and was a joint effort by the haemodialysis staff and the unit for infection prevention and control. It included reviewing the work methods and work space, observations on compliance with standard precautions and handling of the vascular access, creating a checklist and a designated kit for handling the vascular access and prospective surveillance of access-related infections.

Results: During a nine-year period, the haemodialysis units A and B treated 4471 and 7547 patients (mean number of patients per year: 497 (range 435-556) and 839 (range 777-1055), respectively). For most patients, the procedure was done through an arteriovenous fistula (66.7\%, range 50.3-81.5\%). The access-related infection rate decreased significantly in both haemodialysis units: from 3 to $0.9 \%$ (trend: $p<0.05$, linear regression: $p<0.001$ ) in Unit $A$ and from 0.9 to $0.2 \%$ (trend: $\mathrm{p}<0.05$, linear regression: $p=0.01$ ) in Unit $\mathrm{B}$.
\end{abstract}

Conclusions: An intervention which included introduction of a checklist and designated kit, together with ongoing surveillance and feedback, resulted in a significant decrease in the access-related infection rates in both haemodialysis units.

Keywords: Access-related infections, Haemodialysis, Intervention, Surveillance, Checklist

\section{Background}

Infection is the second leading cause of death in haemodialysis patients [1]. Bloodstream infections are a common cause for morbidity and mortality in these patients, many associated with the presence of a vascular access. The incidence of access-related bacteremia is estimated at 0.9-2.0 episodes per one catheter year [2]. The type of vascular access is a critical risk factor for bacteremia. The risk for an access-related bacteremia with a tunnelled catheter is ten times higher than with an arteriovenous (AV) graft and twenty times higher than with an AV fistula [3]. Surveillance is known as an important factor in preventing access-related bacteremia in haemodialysis

\footnotetext{
* Correspondence: Benenson@hadassah.org.il

²Department of Clinical Microbiology and Infectious Diseases, Hadassah Hebrew University Medical Center, POB 12000, 9112001 Jerusalem, Israel Full list of author information is available at the end of the article
}

patients [4]. In 2009, the Centers for Disease Control and Prevention $(\mathrm{CDC})$ published a list of nine core intervention components for dialysis bloodstream infection prevention including hand hygiene and vascular access observations, staff and patient education, disinfection techniques and surveillance [5]. We present the first 9 years of our intervention and surveillance program in two haemodialysis units in a tertiary care center in order to decrease the accessrelated infection rates.

\section{Methods \\ Setting \\ Our intervention and surveillance program was carried out at the Hadassah-Hebrew University Medical Center, Jerusalem, Israel, an 800 bed urban academic tertiary re- ferral center. It included two haemodialysis units under supervision of the nephrology department of Hadassah: the in-hospital 15-station unit of Hadassah Ein-Kerem,}


treating inpatients and outpatients (Unit A), and the 17station outpatient only unit (Unit B). Only outpatients were included in our study. The patient population of Unit A is comprised of patients with a high degree of comorbidities, including oncologic and hematologic patients. Unit B treats more ambulatory patients with less comorbidities (younger and with a better functional status). On December 2015, Unit B was transferred to a new facility within the hospital, increasing its capacity for haemodialysis patients from 15 to 18 stations and allowing it to handle more complex patients.

\section{Data collection}

Since January 1st 2009, a nursing staff member of each unit collected data prospectively about each of the following events: hospitalization, antibiotic treatment, positive blood culture, vascular exit site infection or catheter exchange due to infection. For each event, data was collected about the type of event, type of vascular access, reason for hospitalization (if occurred), blood culture results and vascular access culture results. A census form documenting the number of outpatients treated in each unit and their type of vascular access (AV graft, AV fistula or tunnelled catheter) was completed in the first two work days of each month.

\section{Identification of access-related infection}

At the end of each month, each event was assessed by the program's infectious disease specialist (SB) together with the program's infection control nurse (IG) to determine the presence or absence of access-related infection. The infectious disease specialist and infection control nurse were not involved in routine patient care. They reviewed all clinical, imaging, laboratory and microbiology results and based their evaluation on the CDC criteria for nosocomial infections [6]. Briefly, vascular access exit site infection was defined when pus, redness or swelling of the access site was present without a bloodstream infection that can be related to the vascular access. Bloodstream infection was defined as related to the vascular access when no other source for the bloodstream infection was found and considered secondary when a primary source of infection was present. Both exit site infection and access-related bloodstream infection, were considered as an access-related infection. In the presence of infection, we manage the patient and the catheter according to the Infectious Diseases Society of America (IDSA) clinical practice guidelines for the diagnosis and management of intravascular catheter-related infection [7].

\section{Intervention}

At the beginning of the intervention and surveillance program, infection control staff (nursing and medical) reviewed the working methods in both haemodialysis units. This review included multiple observations examining compliance with standard precautions, handling the vascular access and evaluation of the workspace. Recommendations and guidelines for improvement were issued accordingly. These included: 1) Creating a checklist for handling of the vascular access through all the steps during the haemodialysis session (Table 1). 2) Designing a structured kit, based on the checklist, containing all necessary equipment and composed of three components: a) dressing change; b) connecting the vascular access and c) disconnecting the vascular access. The kit was arranged according to the flow of procedures during the haemodialysis session (Table 2). 3) Emphasizing the advantage in performing shunt operations to minimize the number of patients doing dialysis through a tunnelled central catheter. 4) Establishing staff and patient education protocols. 5) Implementation of a monitoring and reporting system for infectious episodes. 6) Presenting the data to haemodialysis units nursing and medical staff quarterly and annually and discussing in periodical meetings. During the implementation of the intervention and periodically thereafter, the infection prevention team (nursing and medical) reviewed the working methods in both haemodialysis units. This included observations on the process of handling the vascular access.

\section{Analysis}

Access-related infections per 100 patient months were calculated for each unit, both total and per specific access type. In order to assess changes in infection rates over time we examined whether there was a trend over the years (Mann-Kendall test) and also examined the continuous data in a linear regression model. In order to examine whether access types were associated with infection rates, we generated a linear regression model including access types. All analyses were performed with WINPEPI (Abramson, J.H. WINPEPI updated: computer programs for epidemiologists, and their teaching potential. Epidemiologic Perspectives \& Innovations 2011, 8:1).

\section{Results}

During a nine-year period (January 1st 2009 - December 31st 2017), Units A and B treated 4471 and 7547 patients (mean number of patients per year: 497 (range 435-556) and 839 (range 777-1055), respectively). For the majority of patients, the haemodialysis access was through an AV Fistula (66.7\%, range 50.3-81.5\%, Fig. 1a and b).

While the rate of patients with an AV fistula did not change significantly during the study period, there was a decrease in the rate of graft usage parallel to an increase in tunnelled catheter usage (Fig. 1a, Fig. 1b). 
Table 1 Checklist for handling vascular haemodialysis access

\section{Dressing change}

Perform hand hygiene*

Wear a surgical mask (nurse and patient)

Wear non-sterile disposable gloves

Remove the dressing from the catheter without touching adjacent skin

Remove gloves and perform hand hygiene

Open the dressing change kit

Pour 2\% chlorhexidine solution into a bowl

Perform hand hygiene and put on sterile gloves

Place three swabs in the chlorhexidine bowl

Disinfect the exit site thrice, once with each swab; wait for the area to dry

Wrap the catheter hubs with a sterile gauze

Cover the exit site with a sterile dressing

Remove gloves and perform hand hygiene

Connecting the patient to the haemodialysis machine (immediately after dressing change)

Open the kit for connecting the patient

Pour 2\% chlorhexidine solution into a bowl

Wear sterile gloves

Put gauze pads in the chlorhexidine bowl

Place a sterile towel beneath the catheter

Clean the catheter hubs with the gauzes

Draw dead space from each lumen

Connect the haemodialysis tubing

Remove gloves and perform hand hygiene

Disconnecting the patient from the haemodialysis machine

Perform hand hygiene and put on non-sterile gloves

Wear a surgical mask (nurse and patient)

Return blood from the machine

Measure blood pressure

Remove gloves and perform hand hygiene

Open the kit for disconnecting the patient

Pour $2 \%$ chlorhexidine solution into a bowl

Perform hand hygiene and put on sterile gloves

Put gauze pads in the chlorhexidine bowl

Place a sterile towel beneath the catheter

Clean the catheter hubs with the chlorhexidine gauzes

Inject saline and heparin into the catheter

Put sterile caps on the catheter hubs

Put the designated dressing around the catheter hubs

Remove gloves and perform hand hygiene
Table 2 Content of the haemodialysis access handling kit (all material sterile in the kit)
Dressing change
1 set sterile gloves
3 clean swabs
1 empty bowl
Gauzes

Yes/No Tagaderm / Hypodress bandages

Yes/No Connecting the patient to the haemodialysis machine

Yes/No (following the dressing change)

Yes/No Two $5 \mathrm{ml}$ syringes

Yes/No 1 set sterile gloves

Yes/No 1 sterile towel

1 empty bowl

Yes/No Gauzes

Yes/No Disconnecting the patient from the haemodialysis machine

Yes/No 1 empty cup

1 set sterile gloves

1 sterile towel

Yes/No

Yes/No

Yes/No

Yes/No

Yes/No

Yes/No

$\mathrm{Yes} / \mathrm{No}$

Yes/No

Yes/No

Yes/No

Yes/No

Yes/No

Yes/No

Yes/No

Yes/No

Yes/No

Yes/No

Yes/No

Yes/No

Yes/No

Yes/No

Yes/No

Yes/No

Yes/No

* Hand hygiene - Hand disinfection using alcohol-based hand rub
Gauzes

1 designated dressing for the catheter hubs

Tagaderm / Hypodress bandages

2 sterile caps for the catheter hubs

Overall, 938 and 1061 events were reported in the A and $\mathrm{B}$ haemodialysis units by the nursing staff during the study period (mean number of events per year: 104 and 118 respectively). The rates of hospitalization, antibiotic usage, bacteremia episodes and access- related infections are shown in Table 3.

During the study period, the access-related infection rate decreased significantly in the two haemodialysis units: from 3 to $0.9 \%$ (trend: $p<0.05$, linear regression: $p<0.001$ ) in Unit $\mathrm{A}$ and from 0.9 to $0.2 \%$ (trend: $\mathrm{p}<0.05$, linear regression: $p=0.01$ ) in Unit B (Fig. 2). The rates of access-related infections were higher in patients using a tunnelled catheter as compared to an AV graft or an AV fistula (Fig. 3a and b, trend: $p<0.05$, linear regression: $p=0.01$ ). The isolation of the different pathogens is presented in Table 3. Pathogens were isolated in 65/85 (76.5\%) and 31/41 (75.6\%) accessrelated infections in the $\mathrm{A}$ and $\mathrm{B}$ units respectively. The events in which pathogens were not found represent access-exit site infections.

\section{Discussion}

We demonstrated a significant decrease in the rate of access-related infections in two dialysis units during a nine-year period, by implementing an intervention and surveillance program. The rates decreased even though the usage of tunnelled catheters increased. We believe that the main reason leading to this change was the 

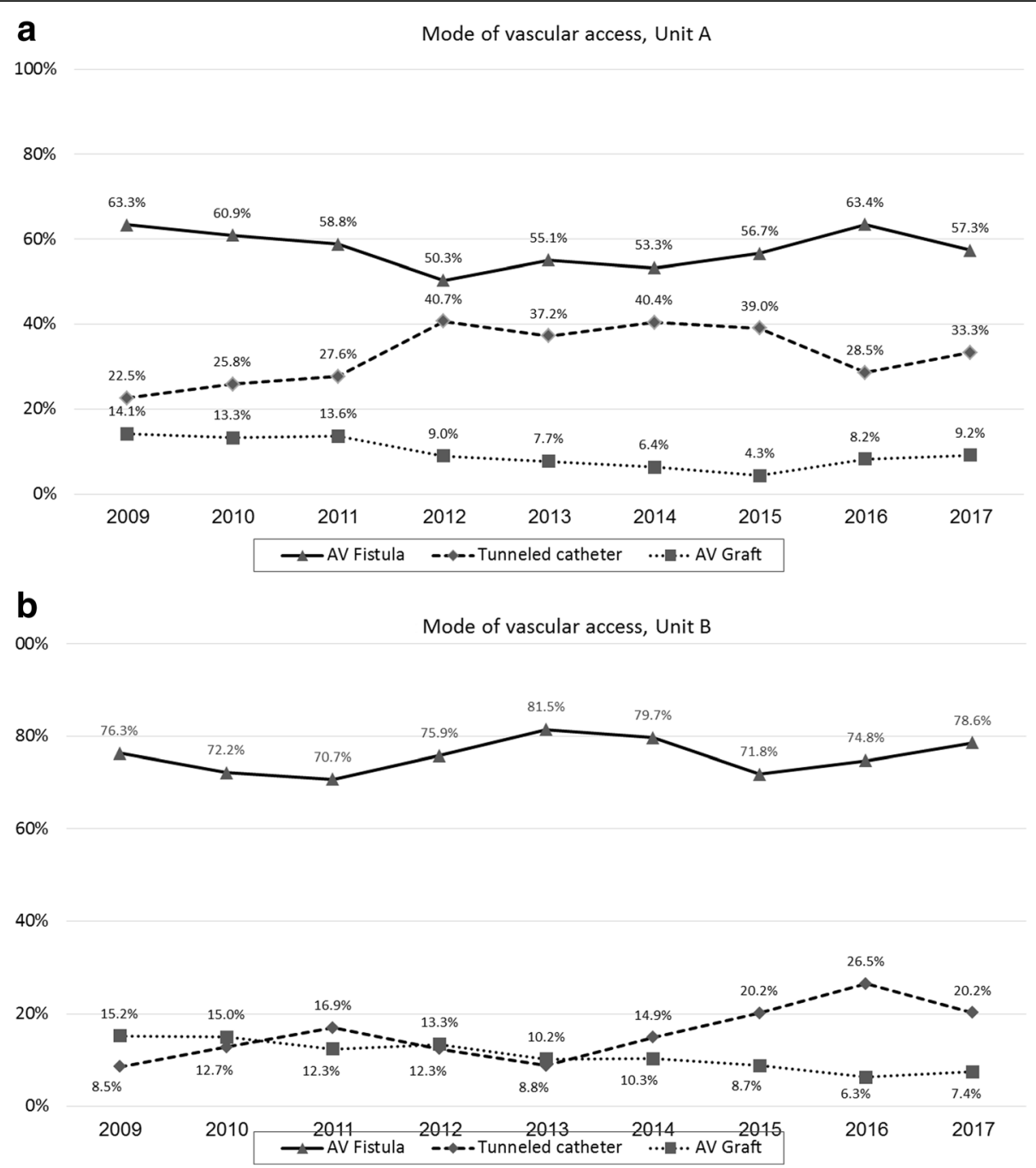

Fig. 1 a, b The proportions of patients with each mode of vascular access (tunnelled catheter, AV graft or fistula) in Unit A (a) and Unit B (b) units. Summarized proportions could exceed $100 \%$ since some patients had both a tunnelled catheter and an AV fistula or graft in the same time until the fistula or graft were fully operational

evaluation of work methods and work space, and following changes in handling the vascular access during the whole dialysis session. Establishing a checklist led to designing a structured kit containing all the equipment needed to perform the procedures, arranged according to the order of steps during the dialysis session. We believe that the implementation of an intervention and surveillance program in which the dialysis units' nurses were involved, and the resulting increased awareness of the staff to the prevention of access-related infections contributed to the decrease in access-related infections as well. The -related infection rate corresponds with the mean access-related infection rates reported in the $\mathrm{Na}$ tional Healthcare Safety Network (NHSN) Dialysis Event Surveillance Report for 2014, as 1.93\% [8].

The ability to reduce central line associated bloodstream infection (CLABSI) by implementing a quality and improvement intervention in intensive care units has been demonstrated previously [9]. This approach was also applicable and effective in haemodialysis patients $[4,10]$. Our study emphasizes the need for checklists and bundles for handling the vascular access in haemodialysis patients, an approach that has been implemented mostly in intensive care unit patients but is less reported in haemodialysis units [11, 12]. Establishing this checklist also guided us in design and use of a dedicated kit containing all of the necessary equipment for handling the vascular access during the whole haemodialysis session, including dressing of the vascular access. Using a readymade kit reduces the need to gather equipment for the procedure manually and has been shown by Pronovost et-al to decrease CLABSI rates in intensive care units [13]. To our knowledge, this approach has not been applied yet in haemodialysis units. 
Table 3 Characteristics of the events* in both dialysis units during a nine-year study period

\begin{tabular}{|c|c|c|}
\hline & Unit A & Unit B \\
\hline & N (\%) & N (\%) \\
\hline Total events & 938 & 1061 \\
\hline Hospitalizations & $700(75)$ & $725(68)$ \\
\hline Treated with antimicrobials & $648(69)$ & $747(70)$ \\
\hline Bacteremia episodes & $146(16)$ & $121(11)$ \\
\hline Access exit site infection ${ }^{\&}$ & 27 (3) & $13(1)$ \\
\hline Access-related infections & $85(9)$ & $41(4)$ \\
\hline Pathogens isolated ${ }^{\#}$ & $65(76)$ & $31(76)$ \\
\hline Enterobacteriaceae & $30(46)$ & $8(26)$ \\
\hline Pseudomonas aeruginosa & $8(12)$ & $1(3)$ \\
\hline Staphylococcus aureus & $11(17)$ & $15(48)$ \\
\hline Other gram positive cocci & $13(20)$ & $6(19)$ \\
\hline Other $\$$ & $3(5)$ & $1(3)$ \\
\hline
\end{tabular}

* Events - hospitalization, antibiotic treatment, positive blood culture, vascular exit site infection or

catheter exchange due to infection

${ }^{\&}$ Access exit site infection - tunnelled catheter, $21 / 27(78 \%)$ in unit $A$ and $11 /$ $13(85 \%)$ in unit B; AV graft and AV fistula, each, $3 / 27$ (11\%) in unit A and 1/13 $(8 \%)$ in unit B

\# Pathogens could be isolated either from blood culture or exit site infection

\$ Other - unit A, 2 candida sp. and 1 polymicrobial; unit B, 1 anaerobe

Another tool for the prevention of catheter related infection is the use of antibiotic lock at the end of each haemodialysis session. Some randomized controlled studies and meta-analyses have been done assessing the role of antibiotic locks for the prevention of accessrelated infections in haemodialysis patients and showed a reduction in bacteremia rates. However, most had a short duration of follow-up. So long-term benefit or loss of efficacy, development of antimicrobial resistance, or other adverse effects could not be evaluated [14]. Additionally, this practice is not part of the current guidelines [7]. In our institution, we use antibiotic lock as salvage therapy only, in patients with complicated access-related infections.

The decrease in access-related infections demonstrated in our work occurred despite an increase in the proportion of patients using tunnelled central venous catheters. It is known that tunnelled dialysis lines are prone to get infected about 20 times more often than AV fistulas and ten times more often than intravenous grafts [3]. The increased proportion of patients using tunnelled lines in our dialysis units is not consistent with trends of vascular access types in haemodialysis patients [15]. This unestimated effect occurred despite our efforts to increase the proportion of patients using AV fistula or graft. The increase in the proportion of patients using tunnelled lines might be explained by the fact that our units are hospital based, allowing for increased medical supervision, and therefore treat more complicated and elderly patients that are not always candidates for an AV fistula operation.

Our study has several limitations. We did not collect demographic and clinical data about our haemodialysis patient population; hence we could not compare the patients' characteristics in the 2 units. Even though, the effect of our surveillance and intervention program remains significant and consistent over time in both haemodialysis units. Additionally, we relied on events reporting done by the haemodialysis nursing staff. However, we believe that our surveillance was sensitive enough since the reports included not only bloodstream infections but also fever episodes, antibiotic treatments started and hospitalizations.

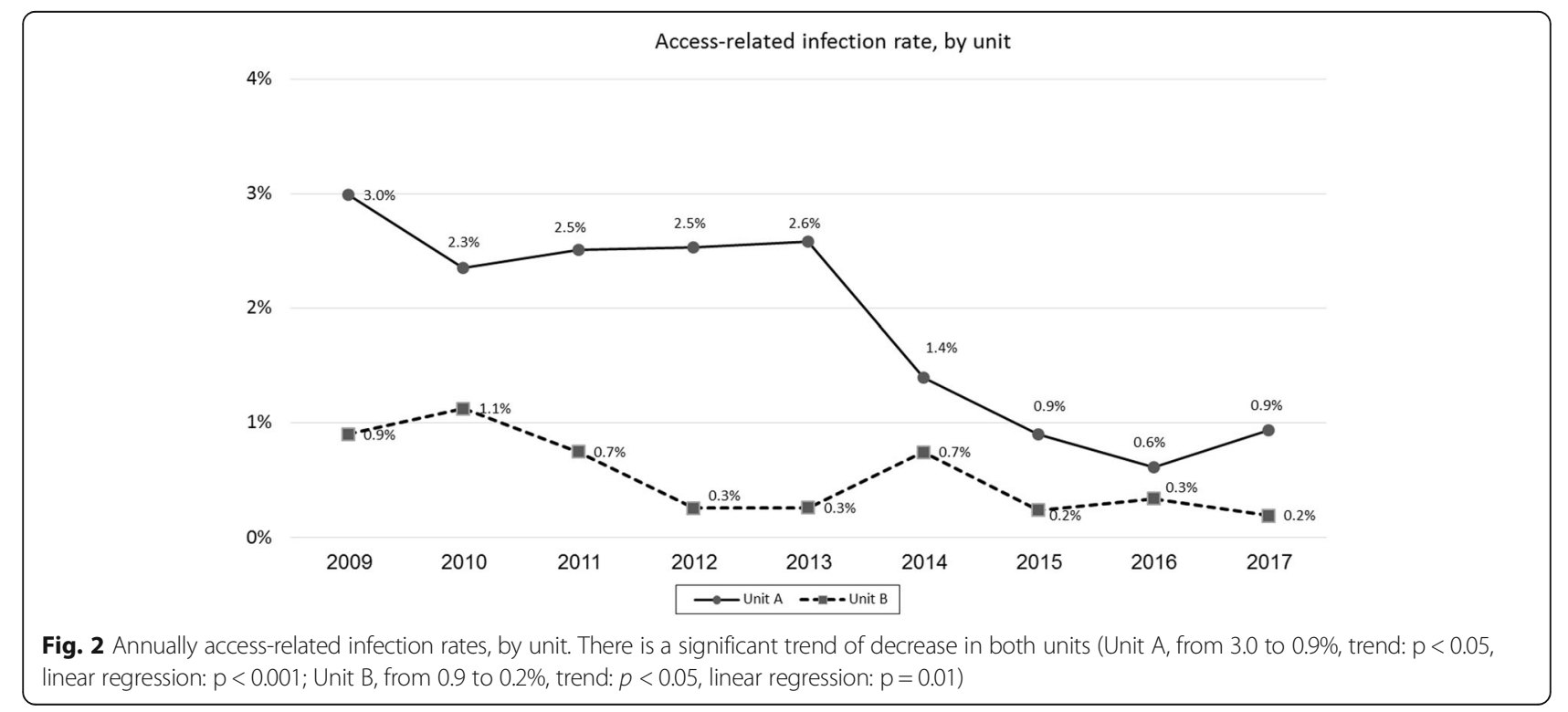




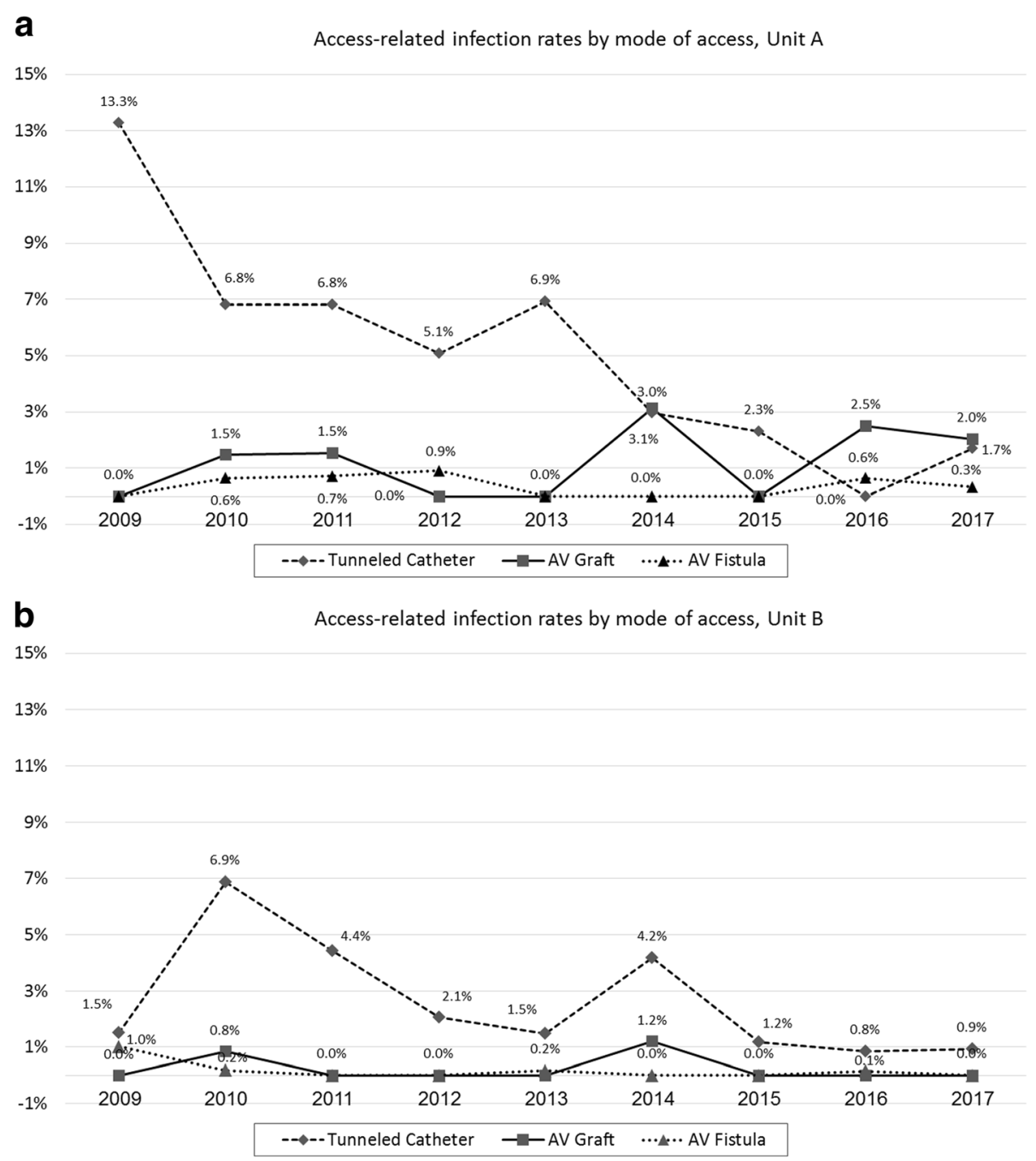

Fig. $\mathbf{3}$ a, b Annually access-related infection rates by mode of vascular access (tunnelled catheter, AV graft or fistula) and unit; Unit A (a), Unit B (b)

\section{Conclusions}

In conclusion, we demonstrated that by implementing an intervention and surveillance program and by using a dedicated checklist and readymade kit for handling the vascular access we were able to significantly lower the access-related infection rates, even in the presence of a high proportion of tunnelled central venous catheters.

\section{Abbreviations}

AV: Arteriovenous; CDC: Centers for Disease Control and Prevention;

CLABSI: central line associated bloodstream infection

\section{Acknowledgements}

Not applicable.

\section{Authors' contributions}

IG This author participated in data collection, performed the data analysis, interpreted the data, wrote the manuscript and has approved the publication of this version. He is accountable for the accuracy and integrity of the work. IG This author participated in the data collection, interpreted the data, writing of the manuscript and has approved the publication of this version. MJC This author participated in the data collection, data analysis, writing of the manuscript and has approved the publication of this version. CS This author participated in study design, oversight and manuscript preparation and has approved the publication of this version. AEM This author participated in study design, oversight and manuscript preparation and has approved the publication of this version. MDE This author Participated in study design, oversight and manuscript preparation and has approved the publication of this version. SB This author conceived and designed the study, participated in data collection, performed the data analysis, interpreted the data, and wrote the manuscript. He has approved the publication of this version and is accountable for the accuracy and integrity of the work.

\section{Funding}

No funding was given for this study.

\section{Availability of data and materials}

The datasets used and/or analysed during the current study are available from the corresponding author on reasonable request.

\section{Ethics approval and consent to participate}

The study was done in our institution as part of quality improvement. Ethics approval is not necessary for this type of quality improvement project, which is part of the everyday clinical attention our patients receive. 


\section{Consent for publication}

Not applicable.

\section{Competing interests}

The authors declare that they have no competing interests.

\section{Author details}

'Department of Nephrology, Hadassah-Hebrew University Medical Center, Jerusalem, Israel. ${ }^{2}$ Department of Clinical Microbiology and Infectious Diseases, Hadassah - Hebrew University Medical Center, POB 12000, 9112001 Jerusalem, Israel.

Received: 14 February 2019 Accepted: 10 June 2019

Published online: 18 June 2019

\section{References}

1. Collins AJ, Foley RN, Gilbertson DT, Chen SC. United States renal data system public health surveillance of chronic kidney disease and end-stage renal disease. Kidney Int Suppl. 2015;5:2-7.

2. Allon M. Dialysis catheter-related bacteremia: treatment and prophylaxis. Am J Kidney Dis. 2004;44:779-91.

3. Tokars Jl, Miller ER, Stein G. New national surveillance system for hemodialysis-associated infections: initial results. Am J Infect Control. 2002; 30:288-95.

4. George A, Tokars JI, Clutterbuck EJ, Bamford KB, Pusey C, Holmes AH. Reducing dialysis associated bacteraemia, and recommendations for surveillance in the United Kingdom: prospective study. BMJ. 2006;332:1435.

5. Centers for Disease Control and Prevention. CDC approach to BSI prevention in dialysis facilities. 2006. Retrieved from http://www.cdc. gov/ dialysis/prevention-tools/core-interventions. Accessed 1 Aug 2018.

6. Klevens RM, Edwards JR, Richards CL Jr, Horan TC, Gaynes RP, Pollock DA, et al. Estimating health care-associated infections and deaths in US hospitals, 2002. Public Health Rep. 2007;122:160-6.

7. Mermel LA, Allon M, Bouza E, Craven DE, Flynn P, O'Grady NP, et al. Clinical practice guidelines for the diagnosis and management of intravascular catheter-related infection: 2009 update by the Infectious Diseases Society of America. Clin Infect Dis. 2009:49:1-45.

8. Nguyen DB, Shugart A, Lines C, Shah AB, Edwards J, Pollock D, et al. National Healthcare Safety Network (NHSN) dialysis event surveillance report for 2014. Clin J Am Soc Nephrol. 2017;12:1139-46.

9. Blot K, Bergs J, Vogelaers D, Blot S, Vandijck D. Prevention of central lineassociated bloodstream infections through quality improvement interventions: a systematic review and meta-analysis. Clin Infect Dis. 2014;59: 96-105.

10. Patel PR, Yi SH, Booth S, Bren V, Downham G, Hess S, et al. Bloodstream infection rates in outpatient hemodialysis facilities participating in a collaborative prevention effort: a quality improvement report. Am J Kidney Dis. 2013;62:322-30

11. Sacks GD, Diggs BS, Hadjizacharia P, Green D, Salim A, Malinoski DJ. Reducing the rate of catheter-associated bloodstream infections in a surgical intensive care unit using the Institute for Healthcare Improvement Central Line Bundle. Am J Surg. 2014;207:817-23.

12. Ista E, van der Hoven B, Kornelisse RF, van der Starre C, Vos MC, Boersma E, et al. Effectiveness of insertion and maintenance bundles to prevent central-lineassociated bloodstream infections in critically ill patients of all ages: a systematic review and meta-analysis. Lancet Infect Dis. 2016;16:724-34.

13. Pronovost P, Needham D, Berenholtz S, Sinopoli D, Chu H, Cosgrove S, et al. An intervention to decrease catheter-related bloodstream infections in the ICU. N Engl J Med. 2006;28(355):2725-32.

14. Miller LM, Clark E, Dipchand C, Hiremath S, Kappel J, Kiaii M, et al. Hemodialysis tunneled catheter-related infections. Can J Kidney Health Dis. 2016. https://doi.org/10.1177/2054358116669129.

15. Pisoni RL, Zepel L, Port FK, Robinson BM. Trends in US vascular access use, patient preferences, and related practices: an update from the US DOPPS practice monitor with international comparisons. Am J Kidney Dis. 2015;65: $905-15$

\section{Publisher's Note}

Springer Nature remains neutral with regard to jurisdictional claims in published maps and institutional affiliations.

\section{Ready to submit your research? Choose BMC and benefit from:}

- fast, convenient online submission

- thorough peer review by experienced researchers in your field

- rapid publication on acceptance

- support for research data, including large and complex data types

- gold Open Access which fosters wider collaboration and increased citations

- maximum visibility for your research: over $100 \mathrm{M}$ website views per year

At BMC, research is always in progress.

Learn more biomedcentral.com/submissions 